\title{
Probing Membrane Nanodomain Organization with Single Particle Tracking via Photoactivated Localization Microscopy (spt-PALM)
}

\author{
Yerim Lee ${ }^{1,2}$, Carey Phelps ${ }^{1,2}$, Tao Huang ${ }^{1,2}$, Barmak Mostofian ${ }^{1,2}$, Daniel Zuckerman ${ }^{1,2}$ and Xiaolin \\ $\mathrm{Nan}^{1,2,3, *}$ \\ 1. Department of Biomedical Engineering, Oregon Health and Science University, Portland, OR, USA. \\ 2. OHSU Center for Spatial Systems Biomedicine, Oregon Health and Science University, Portland, OR, \\ USA. \\ ${ }^{3 .}$ Knight Center for Cancer Early Detection Advanced Research, Oregon Health and Science University, \\ Portland, OR, USA. \\ * Corresponding author: nan@ohsu.edu
}

Accumulating evidence has implicated nanoscopic membrane domains - heterogeneous compartments of the membrane comprising specific subsets of lipids, proteins, and potentially other molecular species - in regulating fundamental cellular processes such as signal transduction, but how these nanodomains organize and interact with various molecules on the membrane remain obscure [1]. This is in a large part due to the small dimensions and often highly dynamic nature of the membrane nanodomains, making it extremely challenging to probe these nanostructures biochemically or microscopically. In recent years, advances in high resolution biological microscopy, most notably superresolution microscopy (SRM) [2], have enabled access to nanoscopic structures and their spatiotemporal dynamics with light. With SRM, it is now possible to characterize the biophysical properties and functional roles of the membrane nanodomains in intact or even live cells [3]. With few exceptions such as caveolae and clathrin-coated pits, however, most putative membrane nanodomains do not even have known markers for fluorescence labeling nor do they exhibit characteristic, morphological contrast under electron microscopy, leaving the presence, properties, and functional relevance of the membrane nanodomains in question.

We reasoned that, for each membrane molecule of interest, its interactions with various membrane nanodomains would manifest as transient changes in diffusion properties. Thus, not only the presence but also important properties of the membrane nanodomains could be probed by following the diffusion of membrane molecules. To this end, we have used single-particle tracking via photoactivated localization microscopy (spt-PALM) [4] to analyze the diffusion properties of KRas, a small GTPase tethered to the inner leaflet of the plasma membrane. Here, KRas is used as a model molecule because it has been previously shown to localize to unidentified membrane nanodomains and form clusters in order to activate its effectors such as Raf [5]. For spt-PALM, KRas is tagged with PAmCherry1, a photoactivatable mCherry with high contrast (brightness after vs before activation) ratio and good single-molecule brightness [6]. As such, KRas diffusion can be analyzed at the single-molecule level even at high expression levels. Through iterative activation and tracking cycles, a large number (10-100k) of singlemolecule KRas diffusion trajectories could be obtained, allowing thorough statistical analysis to define the distinctive diffusion states of KRas and potentially reveal the underlying membrane 'nanodomains' with which KRas is transiently associated.

Using U2OS cells stably expressing PAmCherry1-KRas as a model system, we found that KRas molecules exhibit three distinct diffusion states on the cell membrane, namely a fast state, an immobile state, and a previously unknown intermediate state. By spatially mapping the positions of each diffusion state sampled by a large number of KRas molecules in a short duration, we show that each of the diffusion state of KRas 
is associated with a distinct membrane domain. The immobile and intermediate membrane domains of KRas are both nanoscopic and are spatially correlated; in particular, the immobile domains were each embedded in a larger, intermediate domain. The intermediate and immobile nanodomains also exhibit finite lifetimes, ranging from seconds to minutes, thus indicating the potential presence of multiple types of KRas-associated membrane nanodomains. Analysis of how KRas transitions between the diffusion states further revealed a directional mass flow within the three-state system, suggesting that the membrane domains are also regulating the membrane trafficking of KRas. Together, these results demonstrate the rich information about the underlying membrane organization pertinent to KRas diffusion and trafficking, which has not been obtained previously with other approaches. These novel findings start to paint a clear picture of how KRas operates on the membrane and offers important hints to future studies aiming at defining the KRas-associated membrane nanodomains. The combination of high-through SPT via sptPALM and custom trajectory analysis offers an effective approach to probing the nanodomains associated with other membrane molecules.

\section{References:}

[1] MF Garcia-Parajo et al., J. Cell Sci.

[2] MA Thompson, MD Lew and WE Moerner, Annual Review of Biophysics 41(1) (2012), p. 321.

[3] U Gether et al., Nat. Commun. 8(1) (2017).

[4] S Manley et al., Nat Meth 5(2) (2008), p. 155.

[5] D Abankwa, AA Gorfe and JF Hancock, Semin. Cell Dev. Biol. 18(5) (2007), p. 599.

[6] FV Subach et al., Nat. Methods 6(2) (2009), p. 153.
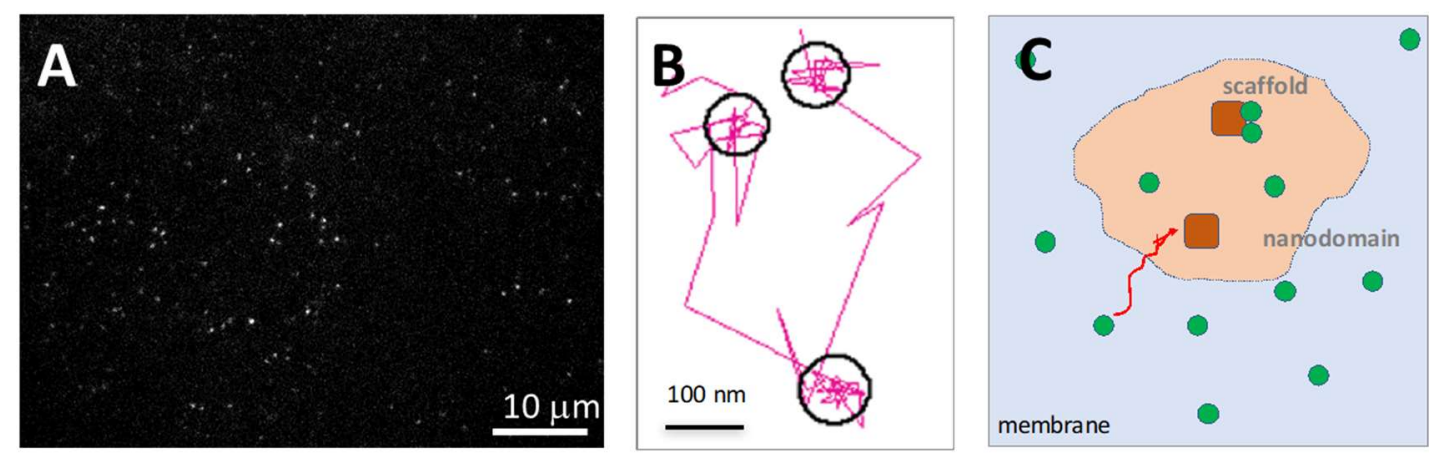

Figure 1. Probing KRas-associated membrane nanodomains with spt-PALM. (A) Snapshot of PAmCherry1KRas molecules in live U2OS cells stochastically activated with low dose $405 \mathrm{~nm}$ illumination and imaged with $561 \mathrm{~nm}$ laser excitation; (B) example single-molecule trajectory of KRas obtained via spt-PALM; (C) proposed nanodomain model of KRas diffusion and trafficking on the cell membrane. Blue: membrane regions conferring fast diffusion; light orange: intermediate membrane nanodomains containing one or more immobile sites (scaffold) that could transiently trap and immobilize KRas. 